\section{Hall-effect device with both voltage leads on one side of the conductor}

\author{
J H J Fluitman \\ Twente University of Technology, PO Box 217, 7500 \\ AE Enschede, The Netherlands
}

Received 28 January 1980

\begin{abstract}
A Hall effect device has been designed and tested with both voltage contacts on one side of the Hall element. The proposed layout lends itself to placing the device on the edge of a substratum (possible application as position detector and recording read head). The response of the device to inhomogeneous magnetic fields has been calculated and the results are in agreement with experiment.
\end{abstract}

\section{Introduction}

In a Hall transducer the voltage contacts are positioned opposite to each other at the edges of the device in the way depicted in figure $1(a)$. In general there is no objection against such a layout, but when the transducer must be placed at the edge of a substratum (e.g. in a position detector or a read head in magnetic recording) there is a difficulty in positioning one

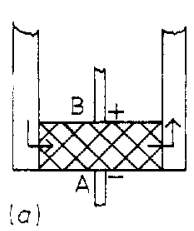

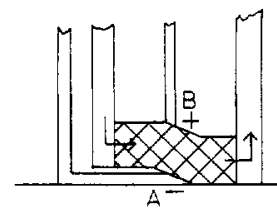

(b)

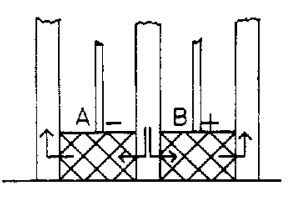

(c)
Figure 1 (a) Traditional layout of a Hall transducer. (b) Layout proposed by Sato et al (1976) in order to position the transducer at the edge of a substratum. (c) Alternative layout proposed in this paper.

of the voltage leads. Then, one has to arrange things, for instance, as proposed by Sato et al (1976) (figure 1(b)) leaving one of the voltage leads right at the edge in a rather awkward and vulnerable position. An attractive alternative for such applications may be the arrangement depicted in figure $1(c)$, consisting in fact of two Hall elements through which currents are lead in opposite directions. If a magnetic field is applied a Hall voltage can be measured between the contacts $\mathrm{A}$ and $\mathrm{B}$. In fact, in a homogeneous field $B$ this Hall voltage has exactly the same magnitude as the voltage detected between opposite contacts. The proposed layout has no contacts at the substratum edge and has the additional advantage that a voltage

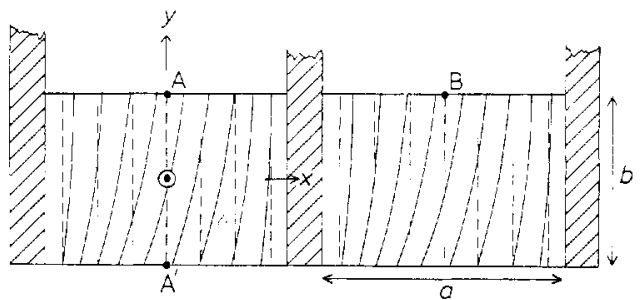

Figure 2 Qualitative picture of the equipotential lines in the cases that a magnetic field is switched on (solid lines) or off (broken lines). In each part the origin of the coordinate system is taken at the centre.

offset at $B=0$ can be trimmed by setting the values of the currents through both parts. In such a way a device can be designed that lends itself to miniaturisation so that the fact that it may be somewhat longer than the traditional layout is hardly a drawback. In figure 2 an impression is given of the potential lines, as they are with and without a magnetic field switched on. The situation is illustrated when the applied field is inhomogeneous in the $y$-direction, as can be expected for the applications mentioned above. We define the Hall potential in any point of the transducer as the difference between the potential with and without a magnetic field applied. In first order the Hall potentials are proportional to $B$. For inhomogeneous magnetic fields which decrease in magnitude for increasing $y$ it can be expected that the voltage $V_{\mathrm{AB}}$ measured between the points $A$ and $B$ will be smaller than between the points $\mathrm{A}$ and $\mathrm{A}^{\prime}\left(V_{\mathrm{AA}}{ }^{\prime}\right)$. Therefore we have to consider the 'quality-factor' $V_{\mathrm{AB}} / V_{\mathrm{AA}}$ ', to see what penalty has to be paid for choosing the proposed layout.

\section{Calculation of the quality factor}

Since we are only interested in the Hall potentials at a few distinct points of the structure, we have chosen the method proposed by Hlásnik and Kokavec (1966) to calculate the potentials. Following their derivation the Hall potential $\phi$ at any point $r^{\prime}$ reads:

$$
\phi\left(\boldsymbol{r}^{\prime}\right)=\frac{1}{2 \pi} \int_{\text {surface }} R_{\mathrm{FF}}(\boldsymbol{B} \times \boldsymbol{J}) . \nabla \ln \left|\boldsymbol{r}-\boldsymbol{r}^{\prime}\right| \mathrm{d} S
$$

with $R_{\mathrm{H}}$ the Hall constant and $J$ the current density at $B=0$. This expression is valid in first order (so that no magnetoresistive effect occurs and the potentials of the current contacts are not influenced by the magnetic field). This expression may be rewritten to read:

$$
\phi\left(\boldsymbol{r}^{\prime}\right)=\frac{R_{\mathrm{H}} I}{2 \pi b t} \int_{-b / 2}^{+b / 2} B(y) G\left(y, x^{\prime}, y^{\prime}, a, b\right) \mathrm{d} y
$$

where $a$ is the length, $b$ the width and $t$ the thickness of the device. It is assumed that $B$ is homogeneous in the $x$-direction, so that the field is represented by $B(y)$. We have calculated the weight function $G$ for position $\mathrm{A}\left(y^{\prime}=b / 2\right)$ and several values of the length/width ratio $a / b$. As can be seen from figure 3 the weight function degenerates to a simple straight line in the limit $a / b \rightarrow \infty$ :

$$
G(y, o, b / 2, a, b)=-2 \pi y / b-\pi
$$

It is also seen that for a value of $a / b=3$ the linear representation is still an acceptable approximation.

From symmetry the weight function for $y^{\prime}=-b / 2$ can easily be derived and is represented in figure 3 as well. In the 


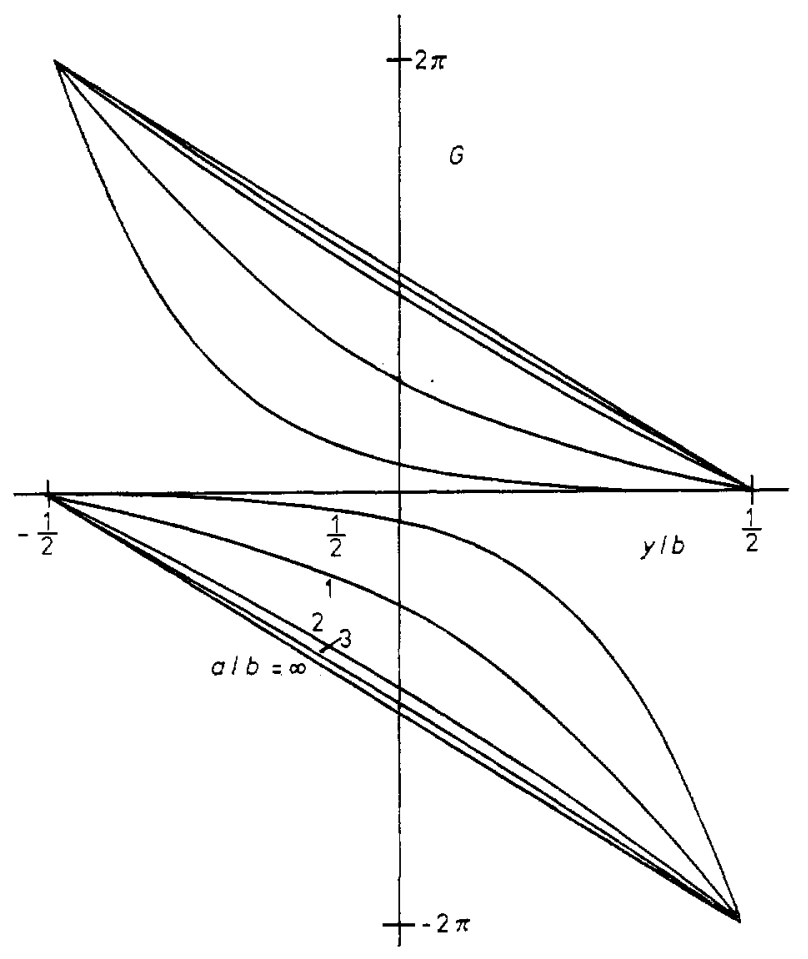

Figure 3 Weight function $G$. The curves at the lower part $(G<0)$ refer to the upper Hall contact (A in figure 2) while the curves at the upper part $(G>0)$ refer to the lower Hall contact ( $\mathrm{A}^{\prime}$ in figure 2). Curves have been calculated for several values of the form factor $a / b$.

limit of linearity it follows:

$$
G(y, o,-b / 2, a, b)=-2 \pi y / b+\pi .
$$

The next step is to introduce an inhomogeneous magnetic field for which we choose

$$
B=B_{0} \exp (-y / c)
$$

with $c$ a measure of the inhomogeneity of $B$. With this field, and for a linear weight function the calculation of the quality

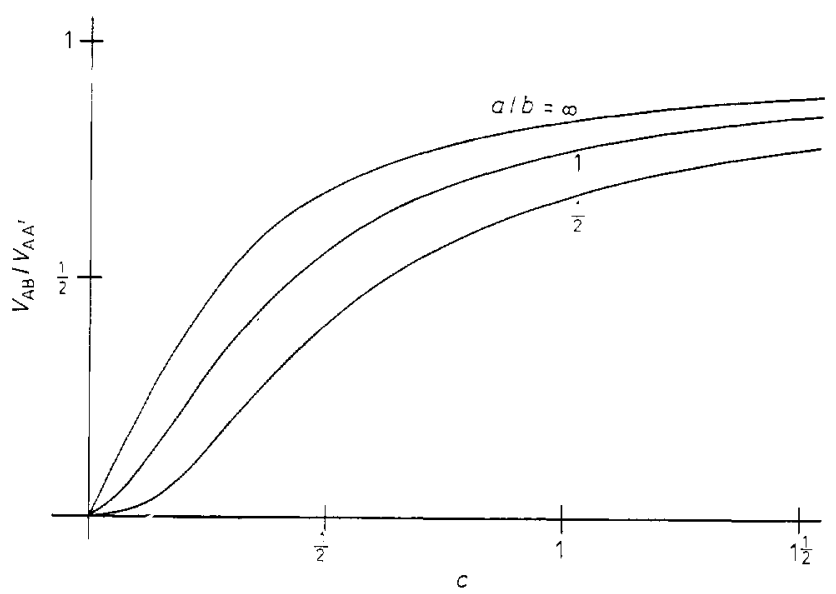

Figure 4 Quality factor $V_{\mathrm{AB}} / V_{\mathrm{AA}}{ }^{\prime}$ as a function of $c$ for the case that an applied inhomogeneous field has the form $B_{0} \exp (-y / c)$. Curves have been calculated for three values of the form factor. factor is straight forward and leads to the result:

$$
V_{A B} / V_{A A^{\prime}}=2 c-\frac{2 \exp (-1 / 2 c)}{\exp (1 / 2 c)-\exp (-1 / 2 c)} .
$$

In figure 4 we have depicted this relation (together with some cases with non-linear weight functions). It can be seen that in case of inhomogeneous fields decreasing with increasing $y$, one must accept a voltage loss. However this need not be disastrous in many practical circumstances.

\section{Comparison with experiment and conclusion}

We have compared the theory with a simple experiment. For the inhomogeneous field we have chosen the fringe field of a magnet with rectangular pole shoes. The field distribution has been measured with a commercial Hall probe which is small compared to the dimension of the magnet. Next we have measured the 'one-sided Hall voltage' on a transducer positioned in a way as depicted in figure 5.

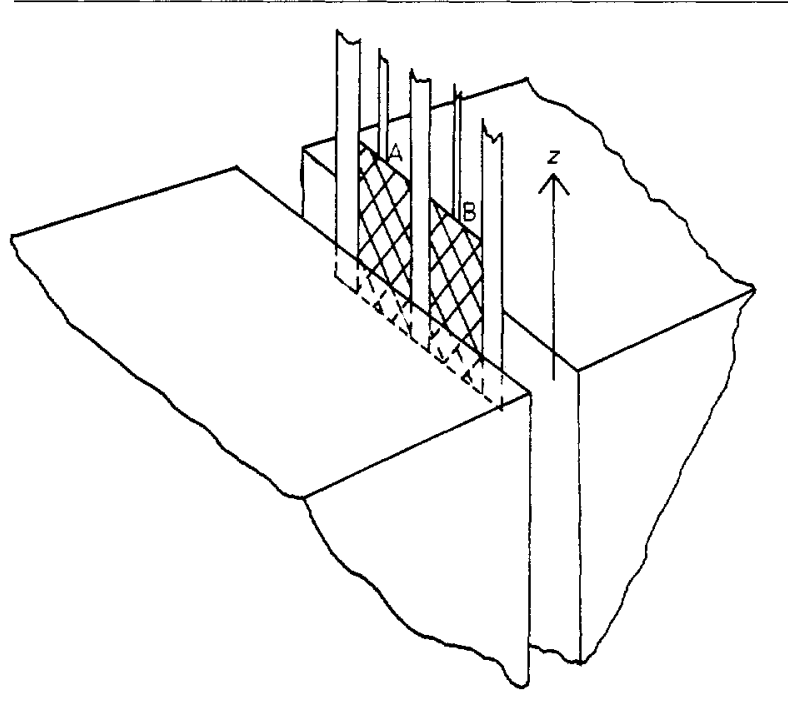

Figure 5 Scheme of the experiment. The Hall transducer is moved in the $z$-direction into the gapfield of a magnet with rectangular pole shoes.

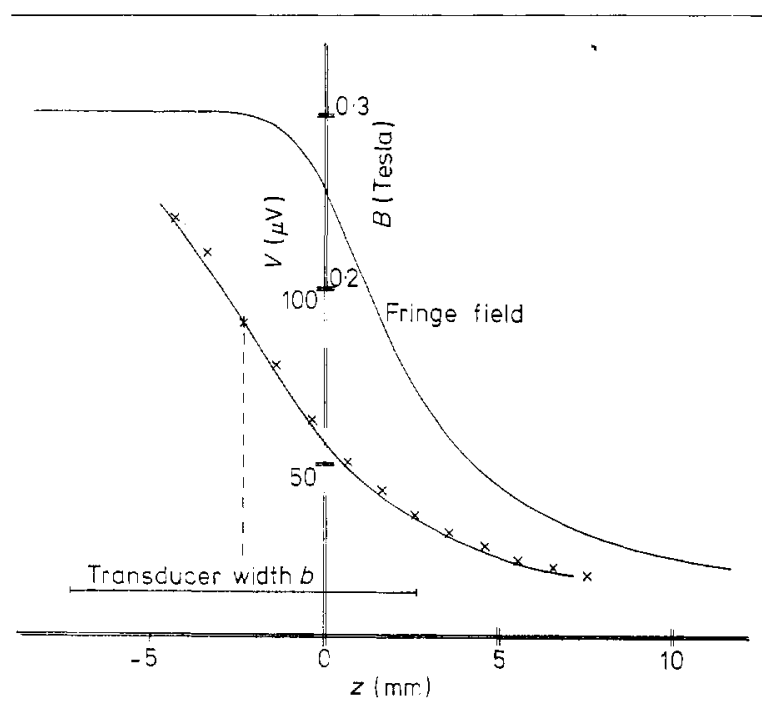

Figure 6 Calculated (lower curve) and measured $(x)$ response of the 'one-sided Hall transducer' in the fringe field (also drawn; upper curve) of the magnet. The position of the gap edge is taken as the origin of the $z$-axis. The response is drawn as a function of the position of the centre of the transducer. 
The transducer consisted of an evaporated bismuth film with dimensions $a=5 \mathrm{~mm}$ and $b=10 \mathrm{~mm}$ on both sides. In figure 6 the measured voltage is shown as a function of the position $z$ of the transducer with respect to the gap edge of the magnet. The agreement with results calculated in the way described in the preceding section is considered as satisfactory so that it is concluded that the 'one-sided Hall transducer' behaves as predicted.

\section{The case of a ferromagnetic strip}

In the preceding sections it has been tacitly assumed that the Hall effect is generated by the Lorentz force on the current carriers. However, in ferromagnetic conductors galvanomagnetic effects may occur as a consequence of an anisotropic conductivity with respect to the direction of magnetisation (Jan 1957). The magnetoresistance of small strips of permalloy, for instance, is utilised in magnetic bubble memories (as a bubble detector) and in magnetic recording devices (as a read head). Especially in the latter case one has made several attempts to circumvent the quadratic character of the magnetoresistivity characteristics in order to produce a transducer with a linear response (leading to elegant solutions like the "barber pole' (Kuijk et al 1975)). An alternative solution to this problem is to exploit the inherently linear Hall effect and to produce a transducer with a layout as proposed in the preceding sections.

For a ferromagnetic strip with the easy axis of magnetisation along the strip-axis the expression for the Hall voltage reads (Wu 1966):

$$
V_{\mathrm{HALL}}=C b / a \sin \phi \cos \phi
$$

with $C$ a constant, $b / a$ the width/length ratio and $\sin \phi=H / H_{\mathrm{K}}$ ( $H_{\mathrm{K}}$ is the effective anistropy field of the strip and $H$ the magnitude of field applied perpendicular to the strip-axis. It must be noted that the Hall effect in ferromagnetic films is generated by fields which are directed in the plane of the film and not perpendicular to it). In materials used in magnetoresistive transducers the value of $C$ is about $3 \%$ of the voltage over the length of the strip. It can be stated that typical values of the Hall voltage are smaller by a factor $b / a$ than typical

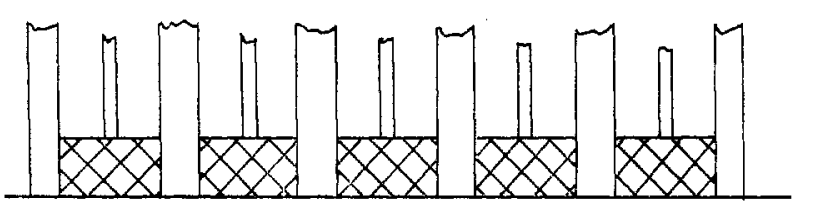

Figure 7 Extended layout of Hall transducer. With such a structure it is possible to read information in parallel, which can be applied as a track sensing facility, for instance.

values of the magnetoresistive voltage. In possible applications as a read head in magnetic recording the proposed layout can be extended along the substratum edge as depicted in figure 7. In this way it is possible to design a transducer with track sensing facilities.

\section{Acknowledgments}

The assistance of Mr T A J Ijzerman and Mr P D Ekkelkamp and other co-workers of our laboratory is kindly acknowledged.

\section{References}

Hlàsnik I and Kokavec J 1966 Hall generator in inhomogeneous field and dipole notion of the Hall effect Solid-St. Electron. 9585
Jan J P 1957 Galvanomagnetic and thermomagnetic effects in metals

Solid St. Phys. 51

Kuijk K E, v Gestel W J and Gorter F W 1975 The barber pole, a linear magneto-resistive head

IEEE Trans. Magn. MAG-11 1215

Sato K, Oi T, Hayashi K, Ketori T and Kotera N 1976 An audio magnetic playback head using InSb thin film Hall elements

J. Acoust. Soc. Japan 3299

Wu ding Ke 1966 Galvanomagnetic effects in Cobalt films Bull. Acad. Sci. USSR 3027 